\title{
Infliximab for the treatment of active scleritis
}

\author{
H. Nida Sen, MD, MHSc ${ }^{\star}$, Amit Sangave, BS $^{\star}$, Keri Hammel, MS ${ }^{\dagger}$, Grace Levy-Clarke, MD*, \\ and Robert B. Nussenblatt, MD \\ "Laboratory of Immunology, National Eye Institute, National Institutes of Health, Bethesda, Md. \\ †EMMES Corporation, Rockville, Md.
}

\begin{abstract}
Objective-This study aimed to evaluate the possible safety and effectiveness of infliximab in patients with active scleritis.

Study Design-Prospective, nonrandomized, open-label pilot study (Protocol No. 04-EI-0065).

Participants-Five patients with active anterior scleritis.

Methods-This single-centre, pilot study of infliximab for the treatment of active anterior scleritis was conducted at the National Eye Institute, National Institutes of Health, between 2003 and 2007. Scleritis patients with active disease who had used at least 1 conventional immunosuppressive agent in the past were included. Primary outcome was a 2-step decrease in scleral inflammation within 14 weeks. Patients received infliximab $(5 \mathrm{mg} / \mathrm{kg}$ ) at baseline, at weeks 2 and 6 , and every 4 weeks through week 30, after which the infusion interval was increased (week 36, 48).
\end{abstract}

Results-All patients met the primary outcome by achieving quiescence of their active scleritis by week 14 with no additional immunosuppressives. However, after 14 weeks 1 patient developed newonset intraocular inflammation that did not respond to reinduction and was terminated from the study. Side effects attributable to infliximab included ear infection with transient decreased hearing, urinary tract infection, lower respiratory tract infection, and facial rash in 1 patient and urinary tract infection, diarrhea, upper respiratory tract infection, nasal congestion and headache, mouth sores, head tremor, and occasional numbness and tingling in extremities in another patient, all of which resolved spontaneously or with appropriate treatment.

Conclusions-Infliximab may be considered as a viable option in treating patients with active scleritis; however, patients should be monitored closely for potentially serious side effects.

\section{Keywords}

infliximab; TNF; scleritis; immunosuppressive therapy

\begin{abstract}
Scleritis is a chronic, painful, and destructive inflammatory disease of the sclera, frequently associated with an infection or underlying systemic disease, and is one of the most challenging conditions to manage in ophthalmology. ${ }^{1}$ In general, noninfectious cases of ocular inflammation are thought to represent immune-mediated disease, possibly triggered by environmental stimuli and mediated primarily by $\mathrm{T}$ cells in patients with an immunogenetic predisposition. $^{2}$
\end{abstract}

Correspondence to H. Nida Sen, MD, National Eye Institute, 10 Center Dr., Bldg. 10, Rm. 10N112, Bethesda, MD 20892; senh@nei.nih.gov.

The authors have no proprietary or commercial interest in any materials discussed in this article. 
Systemic immunosuppressive agents typically utilized in the treatment of scleritis include cyclosporine, azathioprine, methotrexate, cyclophosphamide, and mycophenolate mofetil. In most cases, prompt standard immunosuppressive treatment can reverse the acute inflammatory episode, but side effects can limit these treatments. ${ }^{3-7}$ Treatments are reintroduced or increased as medically indicated, with many patients requiring prolonged and even life-long immunosuppressive treatments. Consequently, the identification of an effective treatment with a safer side effect profile is highly desirable.

Tumor necrosis factor (TNF)-alpha is an inflammatory mediator that plays an important pathogenic role in inflammatory diseases such as rheumatoid arthritis, fistulizing Crohn disease, and ankylosing spondylitis. Ample evidence from animal and human studies implicates TNF as a potentially important mediator in uveitis and scleritis. ${ }^{8-11}$

Infliximab neutralizes the biological activity of human TNF-alpha and has been used in various types of ocular inflammatory diseases, including refractory uveitis and scleritis, with promising results. ${ }^{8,12-15}$

\section{METHODS}

Herein we report the results of a prospective, nonrandomized, open-label pilot study on infliximab in 5 patients with active nonnecrotizing anterior scleritis (National Eye Institute [NEI] Protocol Nos. 04-EI-0065, NCT00075075), done at the NEI between 2003 and 2007. The study was approved by the NEI's International Review Board and monitored by the NEI Serious Adverse Event Review Committee. Informed consent was obtained from all patients.

Primary outcome was defined as (at least) a 2-step decrease in scleral inflammation within 14 weeks of initiating infliximab therapy. Scleritis patients, all of whom had used at least 1 conventional immunosuppressive agent, including steroids, in the past, received infliximab (5 $\mathrm{mg} / \mathrm{kg}$ ) at baseline, at weeks 2 and 6 , and every 4 weeks through week 30, after which its infusion interval was increased (receipt at week 36, 48), and followed up for 6 months after their final infusion for safety ( $8 \mathrm{mg} / \mathrm{kg}$ was allowed for those with increased inflammation). The increased dose was administered at 4-week intervals for 3 infusions and responsive patients then received a regular dose $(5 \mathrm{mg} / \mathrm{kg})$ every 4 weeks for another 3 visits. These patients would receive a total of 12 infusions (10 infusions until week 34, followed by weeks 40 and 52). Scleritis severity was assessed using a composite scoring system that allowed grading of injection and scleral edema (0-4) in each quadrant of the sclera. A 2-step decrease in scleral inflammation within 14 weeks of the baseline was required for a patient to remain in the study. Tapering of immunosuppressives was allowed as of week 2 at the physician's discretion, upon quiescence. Patient termination resulted if scleritis was uncontrolled 14 weeks into the study period, if a recurrence of inflammation after initial control of the active scleritis requiring an increase in concomitant immunosuppressives was seen, or if vision loss was >10 Early Treatment Diabetic Retinopathy Study letters (management according to standard-of-care was resumed). Three terminated participants would end the study.

All patients underwent a careful medical history, thorough physical examination by an internist, review of a detailed health survey, thorough ophthalmic examination at each visit, and purified protein derivative test prior to receiving infliximab in order to rule out tuberculosis. Patients were interviewed regularly at each study visit, for adverse events and medications. All patients had regular blood tests performed at predetermined intervals, which included complete blood count with differential, erythrocyte sedimentation rate, serum electrolytes, hepatic and renal function tests, and urinalysis. Additionally, pregnancy tests for female participants, antinuclear antibody (ANA), and anti-double-stranded DNA tests were carried out regularly. 
Continuous data such as age and time to quiescence are expressed in median values. Paired $t$ test is used for comparing immunosuppressive medication grading before and after the initiation of infliximab.

\section{RESULTS}

The median age of the 5 patients was 34 years (range 21-55). Two patients had human leukocyte antigen B27-associated scleritis, 1 had Cogan Syndrome-associated scleritis, 1 had Wegener's granulomatosis-associated scleritis, and 1 had idiopathic scleritis. With respect to the primary end point, all 5 participants have completed 14 weeks of follow-up and achieved control of their active scleritis within 14 weeks of initiating infliximab therapy. Median time to quiescence was 2 weeks (mean 4; SD 3.6 weeks). Patient 5 developed new-onset intraocular inflammation after 14 weeks. This patient did not respond to reinduction and was terminated from the study. The same patient had a 20-letter drop in best-corrected visual acuity from baseline at week 18 and continued to have a $>15$-letter decrease from baseline in best-corrected visual acuity through week 22 because of a new onset of granulomatous chorioretinitis.

Secondary outcomes included changes in eye pain, redness, photophobia, visual acuity, and amount of concomitant immunosuppressives. Four of the 5 patients showed decreased pain, redness and photophobia, completed the study with no recurrent inflammation, and were able to taper other immunosuppressives. Based on an immunosuppressive grading scale that ranged in score from 0 to 9 for each immunosuppressant ${ }^{16}$ (calculated based on dose in $\mathrm{mg} / \mathrm{kg}$ for each particular immunosuppressive and weighted differently for each immunosuppressive), average immunosuppressive grade showed a nonsignificant decrease from a baseline mean of 3.8-2.6 and 0.5 at weeks 14 and 48 , respectively $(p=0.079)$. Only 3 of 5 successfully tapered prednisone based on Standardization of Uveitis Nomenclature criteria ${ }^{17}$ ( $<10 \mathrm{mg}$ daily). As of the last study visit, 4 of the 5 participants had stable visual acuity (Table 1).

Adverse events attributable to infliximab are reported in Table 1. Infliximab was discontinued in 1 patient secondary to treatment failure (patient 5), but none of the patients discontinued infliximab because of side effects. ANA and anti-double-stranded DNA were negative in all patients before and after infliximab treatment.

\section{CONCLUSIONS}

Infliximab is a chimeric human/murine monoclonal antibody of immunoglobulin G1 isotype with specificity for human TNF. It neutralizes biological activity of TNF-alpha by binding to the soluble and transmembrane forms of TNF-alpha, and inhibits binding of TNF-alpha to its receptors. ${ }^{18}$

Infusion reactions occur in 3\%-17\% of patients and are associated with formation of antibodies to infliximab, and appear to occur more often after the second or third treatments, especially if the last treatment occurred more than 4 months previously. ${ }^{19} \mathrm{We}$ did not observe any infusion reaction in this group of patients. Induction treatment of 3 infusions at short intervals (at 0,2 , and 6 weeks) may reduce the likelihood of development of antibody to infliximab and infusion reactions. Reduction in infusion reactions is possible with concurrent administration of steroids and use of immunosuppressants (azathioprine, 6-mercaptopurine, and methotrexate).

Furthermore, immunosuppressants increase the duration of the response to infliximab. ${ }^{19}$ These factors together may have been responsible for the absence of infusion reactions in our patients, because all were on 1 or more immunosuppressive medications and had received the first 3 infusions at short intervals.

Remarkable responses were reported following infliximab therapy in scleritis and noninfectious uveitis of different etiologies. ${ }^{8,10,11,14,20-22}$ In this study, $80 \%$ of our scleritis 
patients achieved quiescence on treatment and $60 \%$ were able to successfully taper prednisone to $<10 \mathrm{mg}$ daily. None of the patients achieved drug-induced remission based on Standardization of Uveitis Nomenclature criteria, which is defined as drug-free remission; however, 2 patients achieved control of the disease with monotherapy with infliximab.

One patient who was considered a treatment failure had Wegener's granulomatosis; this group of patients tends to pose a management challenge. Although there have been reports of successful use of infliximab in patients with Wegener's, ${ }^{15}$ it has not been our experience. Extensive studies of TNF-alpha gene mutations as predictors of response have led to indeterminate results, and it is unclear whether polymorphisms in the TNF-R1 and TNF-R2 receptors or in various other apoptosis genes have any association with treatment response. 23,24

Adverse events directly attributable to infliximab were observed in $40 \%$ of scleritis patients. We did not observe any embolic side effects with infliximab, in contrast to previous reports. 8,25

Although infliximab helped taper other immunosuppressives, it did not induce remission in any of the patients. Our experience indicates that infliximab may be considered as a viable option in patients with active scleritis; however, patients should be monitored closely for potentially serious side effects.

The significant bias introduced by both patient selection and the observers of uncontrolled studies should be noted; choosing patients who are refractory to other standard immunosuppressives could lead to underestimation of the effectiveness of the drug, whereas unmasking of the observer may lead to overestimation. In general, assessment of infliximab efficacy is hampered by the frequent concomitant use of other drugs and by the mostly short duration of follow-up. Although published results are encouraging, with a prompt and sometimes dramatic response to infliximab observed in many cases, one should be aware of the bias to publish positive findings and failure to disclose therapeutic failures. This bias can only be confidently ruled out by well-designed, randomized, controlled trials.

\section{Acknowledgments}

This work was supported by the National Eye Institute Intramural Research Program.

\section{REFERENCES}

1. McCluskey, PJ.; Wakefield, D. Scleritis and episcleritis. In: Pepose, JS.; Holland, GN.; Wilhelmus, KR., editors. Ocular Infection and Immunity. St. Louis, Mo.: Mosby-Year Book Inc; 1996. p. 642-661.

2. Nussenblatt RB. The natural history of uveitis. Int Ophthalmol 1990;14:303-308. [PubMed: 2249907]

3. Hakin KN, Ham J, Lightman SL. Use of cyclosporin in the management of steroid dependent nonnecrotising scleritis. Br J Ophthalmol 1991;75:340-341. [PubMed: 2043575]

4. Foster CS, Forstot SL, Wilson LA. Mortality rate in rheumatoid arthritis patients developing necrotizing scleritis or peripheral ulcerative keratitis. Effects of systemic immunosuppression. Ophthalmology 1984;91:1253-1263. [PubMed: 6514289]

5. Shah SS, Lowder CY, Schmitt MA, Wilke WS, Kosmorsky GS, Meisler DM. Low-dose methotrexate therapy for ocular inflammatory disease. Ophthalmology 1992;99:1419-1423. [PubMed: 1407973]

6. Sen HN, Suhler EB, Al-Khatib SQ, Djalilian AR, Nussenblatt RB, Buggage RR. Mycophenolate mofetil for the treatment of scleritis. Ophthalmology 2003;110:1750-1755. [PubMed: 13129873]

7. Jabs DA, Rosenbaum JT, Foster CS, et al. Guidelines for the use of immunosuppressive drugs in patients with ocular inflammatory disorders: recommendations of an expert panel. Am J Ophthalmol 2000;130:492-513. [PubMed: 11024423] 
8. Suhler EB, Smith JR, Wertheim MS, et al. A prospective trial of infliximab therapy for refractory uveitis: preliminary safety and efficacy outcomes. Arch Ophthalmol 2005;123:903-912. [PubMed: 16009830]

9. Martin TM, Smith JR, Rosenbaum JT. Anterior uveitis: current concepts of pathogenesis and interactions with the spondyloarthropathies. Curr Opin Rheumatol 2002;14:337-341. [PubMed: 12118164]

10. Di Girolamo N, Visvanathan K, Lloyd A, Wakefield D. Expression of TNF-alpha by human plasma cells in chronic inflammation. J Leukoc Biol 1997;61:667-678. [PubMed: 9201257]

11. Seo KY, Lee HK, Kim EK, Lee JH. Expression of tumor necrosis factor alpha and matrix metalloproteinase-9 in surgically induced necrotizing scleritis. Ophthalmic Res 2006;38:66-70. [PubMed: 16319517]

12. Murphy CC, Greiner K, Plskova J, et al. Neutralizing tumor necrosis factor activity leads to remission in patients with refractory noninfectious posterior uveitis. Arch Ophthalmol 2004;122:845-851. [PubMed: 15197059]

13. Murphy CC, Ayliffe WH, Booth A, Makanjuola D, Andrews PA, Jayne D. Tumor necrosis factor alpha blockade with infliximab for refractory uveitis and scleritis. Ophthalmology 2004;111:352356. [PubMed: 15019389]

14. Joseph A, Raj D, Dua HS, Powell PT, Lanyon PC, Powell RJ. Infliximab in the treatment of refractory posterior uveitis. Ophthalmology 2003;110:1449-1453. [PubMed: 12867408]

15. El-Shabrawi Y, Hermann J. Anti-TNF alpha therapy in chronic necrotizing scleritis resistant to standard immunomodulatory therapy in a patient with Wegener's granulomatosis. Eye 2005;19:1017-1018. [PubMed: 15389262]

16. Buggage RR, Levy-Clarke G, Sen HN, et al. A double-masked, randomized study to investigate the safety and efficacy of daclizumab to treat the ocular complications related to Behçet's disease. Ocul Immunol Inflamm 2007;15:63-70. [PubMed: 17558830]

17. Jabs DA, Nussenblatt RB, Rosenbaum JT. Standardization of Uveitis Nomenclature (SUN) Working Group. Standardization of uveitis nomenclature for reporting clinical data. Results of the First International Workshop. Am J Ophthalmol 2005;140:509-516. [PubMed: 16196117]

18. Knight DM, Trinh H, Le J, et al. Construction and initial characterization of a mouse-human chimeric anti-TNF antibody. Mol Immunol 1993;30:1443-1453. [PubMed: 8232330]

19. Hommes DW, Oldenburg B, van Bodegraven AA, et al. Guidelines for treatment with infliximab for Crohn's disease. Neth J Med 2006;64:219-229. [PubMed: 16929083]

20. Markomichelakis NN, Theodossiadis PG, Pantelia E, Papaefthimiou S, Theodossiadis GP, Sfikakis PP. Infliximab for chronic cystoid macular edema associated with uveitis. Am J Ophthalmol 2004;138:648-650. [PubMed: 15488796]

21. Sfikakis PP, Kaklamanis PH, Elezoglou A, et al. Infliximab for recurrent, sight-threatening ocular inflammation in Adamantiades-Behçet disease. Ann Intern Med 2004;140:404-406. [PubMed: 14996689]

22. El-Shabrawi Y, Hermann J. Anti-tumor necrosis factor-alpha therapy with infliximab as an alternative to corticosteroids in the treatment of human leukocyte antigen B27-associated acute anterior uveitis. Ophthalmology 2002;109:2342-2346. [PubMed: 12466181]

23. Mascheretti S, Hampe J, Kühbacher T, et al. Pharmacogenetic investigation of the TNF/TNF-receptor system in patients with chronic active Crohn's disease treated with infliximab. Pharmacogenomics J 2002;2:127-136. [PubMed: 12049175]

24. Pierik M, Vermeire S, Steen KV, et al. Tumour necrosis factor-alpha receptor 1 and 2 polymorphisms in inflammatory bowel disease and their association with response to infliximab. Aliment Pharmacol Ther 2004;20:303-310. [PubMed: 15274667]

25. Eklund KK, Peltomaa R, Leirisalo-Repo M. Occurrence of pulmonary thromboembolism during infliximab therapy. Clin Exp Rheumatol 2003;21:679. [PubMed: 14611130] 


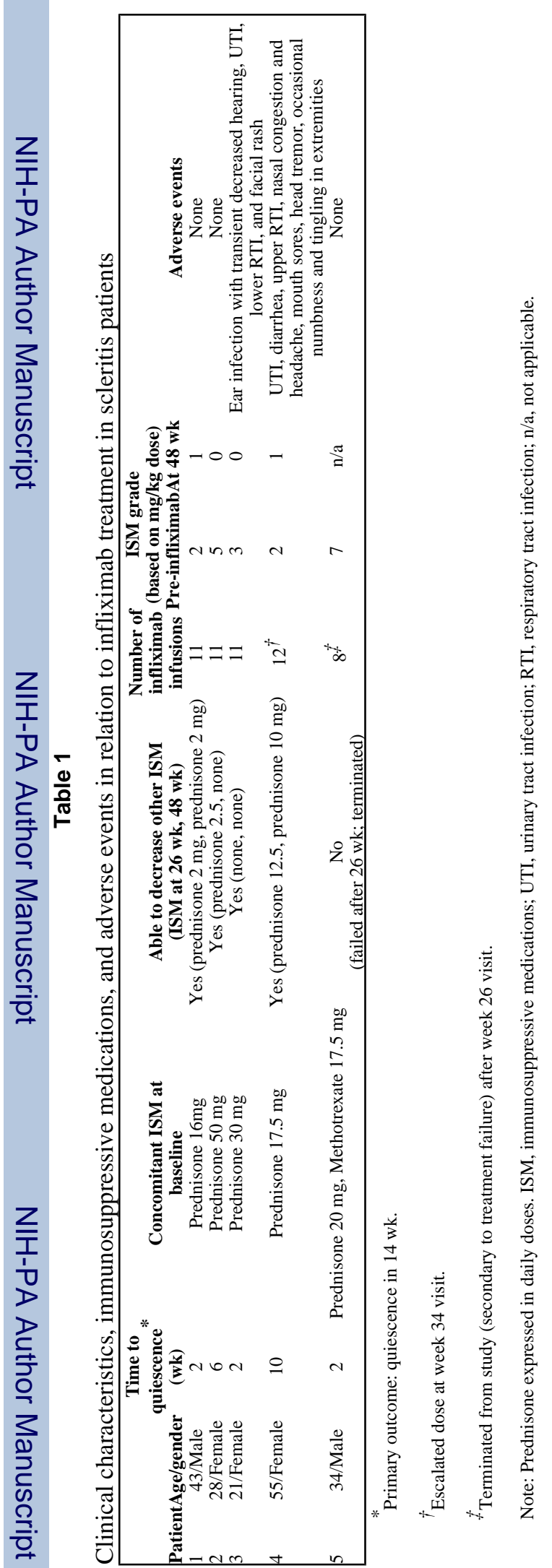

Can J Ophthalmol. Author manuscript; available in PMC 2009 October 31. 